\title{
Ueber ein neues Verfahren zur Darstellung des Kreosots;
}

\author{
von
}

Andreas Cozzi.

(Auszug aus dem Journal de Pharmacie XXIII. 629.)

5o Pfund Theer werden in einer Blase mit Helm erhitz1, und das Produkt der Destillation fängt man in einem cylindrischen zur Hälfte mit Wasser gefïlltem Gefässe auf. Das Produkt ist Essigsäure, Eupion, Paraflin und endlich Kreosot, leicht erkennbar an seinem grösseren specifischen Gewicht als Wasser.

Nach Beendigung dieser ersten Operation wird das noch unreine Kreosot von den andern Produkten mittelst eines Hebers isolirt, und um es besser zu zertheilen, wird es jetzt mit Schwefelsäure, die mit ihrer Gewichtshälfte Wasser verdünnt ist, versetzt. Da das Kreosot sich hier mit einer Flüssigkeit in Berührung findet, die specifisch schwerer ist als es selbst, so steigt es auf die Oberfläche in Form einer Flüssigkeit an ölarliger Consistenz, schwarzer Farbe, die Eiweiss zum Gerinnen bringt und in Alkohol und Essigsäure löslich ist. Dieses Kreosot ist noch mit vielem Eupion vermischt, ich erhitzte es, indem ich es durch eine kochende Mischung von Säure und Wasser gehen liess, und sammelte es in einer weitmündigen bis zum Driltel damit gefiillten Flasche. Es blieb so drei Tage lang mit der Luft in Berührung, die man durch Oeffnen der Flasche mehrmals erneute.

Die Flüssigkeit hatte jetzt eine grössere Consistenz und dunklere Farbe erlangt, sie wurde aus einer durch eine Weigeistlampe erhitzten Retorle einer neuen Destillation unterworfen, und ich erhielt ein röthliches Produkt, welches 
dreimal auf dieselbe Weise behandelt, mir ein Kreosot gab von folgenden Eigenschaften: Es ist wasserhell, von öliger Konsistenz, besitzt in einem hohen Grade die Eigenschaft das Lichts zu brechen, hat einen eigenthümlichen Geruch, einen brennenden Geschmack, ein spec. Gew. von 1,007, kocht bei $203^{\circ}$ R., ist löslich in Essigsäure und Alkobol; $100 \mathrm{Wasser}$ von $20^{\circ} \mathrm{C}$. nehmen $1 \frac{\mathrm{r}}{4} \mathrm{Kreosot}$ auf, es coagulirt das Eiweiss und hat keine Wirkung auf Lackmus und Curcumä. Diese Charaktere sind die des reinen Kreosots.

Der Sauerstoff der atmosphärischen Luft verharzt das Eupion, benimmt ihm dadurch seine Flüchtigkeit, und man wird wahrscheinlich durch einen Strom von Sauerstoffgas oder besser vielleicht durch Zusatz von Schwefelsäure und Manganoxyd dasselbe Resultat erhalten.

Reichen b a ch zeigie die Eigenschaft des Kreosots, thierische Substanzen zu koaguliren, zu erhärlen und so vor Fäulniss zu schützen, und dass es darin Vorzüge besitze vor den besten fäulnisswidrigen Milteln. Er suchte zu beweisen, dass die unmerkliche chemische Wirkung des Kreosots in Wasser aufgelöst, sich vorzïglich darauf beschränkt, eine stärkere Cohäsion thierischer Materien zu bewirken und dadurch einen grössern Widersland gegen von aussen einwirkende Agentien. Was durch Kreosot conservirte thierische Nahrungsmittel betrifft, so befreit man sie von allem Geruch durch mehrmaliges $\Delta$ bwaschen, sie kommen zu ihrem natürlichen Zustande zurück.

Was anatomische Präparate betrifft, so ist bekannt, dass dieselben durch Metallsalze früher oder später eine Veränderung der Farbe erleiden und erhärten. Eine Auflösung von Kreosot ist zum Theil frei von diesen Fehlern. Einige Eintauchungen, eine Injection dieser Flüssigkeit in die Gefässe, reichen für die Conservirung der Substanzen hin. $\mathrm{Zu} \mathrm{dem}$ Vortheile, dass die thierischen Theile durch das Kreosot we- 
nig erhärtet werden, kömmt auch der, dass sie dadurch nicht verunstaltet werden und ihre Structur nicht verlieren.

Ich führe über diesen Gegenstand folgende Versu. che an:

1) Ein Stück Muskelfleisch, welches drei Stunden lang in einer Auflösung von Kreosot gelegen hatte, zeigte sich nach vier Monaten unverändert.

2) Ein Gehirn, welches eben so lange in der Auflösung gelegen hatte, zeigte sich nach funfzelın Tagen olne Veränderung. Es ist daher wahrscheinlich, dass wenn man dem Alkohol, worin man diese weichen Theile aufbewabrt, Kreosot zusetzt, diese nicht so leicht einer Zersetzung unterworfen seyn werden.

3) Ein Vogel und ein Fisch, denen man die Eingeweide und die übrigen weichen Theile ausgenommen, und in deren Höhlungen man Baumwolle gesteckt hatte, die mit Alkohol, der mit wenig Kreosot versetzt, getränkt war, conservirten sich vortrefflich; das Gefieder des ersten blieb unversehrt.

4) Das Kreosot ist bekanntlich ein gutes Auflösungsmittel des Kautschucks, es ist in diesem Betracht dem Aether und den bisher dazu gebrauchten flüchtigen Oelen vorzuziehen. Es hat nicht den Uebelstand, dass es wie diese nach Verflüchligung des Auflösungsmittels Risse hinterlässt, und dass man der Materie jede beliebige Form eindrücken kann, ohne zu befürchten, dass dieselbe durch Austrocknen verändert werde.

5) Schellak, Mastix, Terpentin, Copal und alle Harze lösen sich leicht in Kreosot auf, und die so dargestellten Firnisse haben einen Glanz und eine Durchsichtigkeit weit vorzüglicher, als die mit Alkohol, mit flüchtigen Oelen, oder mit austrocknenden Oelen dargestellten.

6) Das Kreosot ist ein vortreffliches Auflösungsmittel 
des Bernsteins, es bildet damit einen sehr consistenten, zähen und emailartigen Firniss.

7) Viele färbende Materien werden durch Kreosot aufgelöst, und es lässt sich erwarten, dass es auch in der Färberei Anwendung finden wird $\left.{ }^{*}\right)$.

Zweite $\Lambda$ b theilung. Physik.

\title{
Versuche über die Veränderung der Scale der Quecksilber-Thermometer;
}

\author{
von \\ J. N. Legrand.
}

Die Veränderung des Nullpunkts in den QuecksilberThermometern ist eine durch mehre Physiker constatirte Thatsache; es scheint mir aber, dass man bis jetzt die Zeit, wel-

*) In der Abhandlung des Herrn Cozzi findet sich die Stelle, dass ohnerachtet die Darstellung des Kreosots durch die Methoden von Calderini, del Buc und Hübschmann u. a. verbessert sey, dasselbe doch noch theuer zu stehen komme, und die Akademie zu Berlin deshalb eine leichte und wohlfeile Darstellung des Kreosots zum Gegenstand einer Preisfrage gemacht habe. Dieses beruht wahrscheinlich auf einen Trrthum. Es ist uns nicht bekannt, dass die Akademie in Berlin eine derartige Preisfrage aufgegeben hat, wohl aber wurde vor einigen Jahren eine solche von der Hagen-Bucholzschen Stiftung aufgegeben. S, darüber pharmaceut. Zeit. VIII. Jahrg. 1834. S. 312. Es befindet sich daselbst auch die vorzügliche vom Herrn Apothekex J.E. Sim on in Berlin angegebene Methode: Uebrigens wird das Kreosot in mehren chemischen Fabriken in Böhmen in ansehnlicher Menge bereitet, und ist auch von dort zu billigen Preisen zu erhalten.

d. Red. 